Rabaska

Revue d'ethnologie de l'Amérique française

\title{
Benoît Lacroix, O.P.
}

\section{Pietro Boglioni}

Volume 5, 2007

URI : https://id.erudit.org/iderudit/019028ar

DOI : https://doi.org/10.7202/019028ar

Aller au sommaire du numéro

Éditeur(s)

Société québécoise d'ethnologie

ISSN

1703-7433 (imprimé)

1916-7350 (numérique)

Découvrir la revue

Citer ce document

Boglioni, P. (2007). Benoît Lacroix, O.P. Rabaska, 5, 87-99.

https://doi.org/10.7202/019028ar

Ce document est protégé par la loi sur le droit d'auteur. L'utilisation des services d'Érudit (y compris la reproduction) est assujettie à sa politique d'utilisation que vous pouvez consulter en ligne.

https://apropos.erudit.org/fr/usagers/politique-dutilisation/
Cet article est diffusé et préservé par Érudit.

Érudit est un consortium interuniversitaire sans but lucratif composé de l'Université de Montréal, l'Université Laval et l'Université du Québec à Montréal. Il a pour mission la promotion et la valorisation de la recherche. https://www.erudit.org/fr/ 


\section{Portrait \\ Benoît Lacroix, O.P.}

Préparé par Pietro Boglioni

Université de Montréal

En 1995, à l'occasion de son $80^{\circ}$ anniversaire, ses confrères, collègues, anciens élèves et amis offraient au père Benoît Lacroix un hommage extraordinaire : un volume dans lequel cent soixante-treize témoins lui disaient leur reconnaissance et leur admiration. Par touches successives, émergeait de ces témoignages la figure d'un humaniste chrétien accompli, historien, théologien, essayiste, anthropologue, homme de lettres, homme d'Église. Les sept cents pages de ces Dits et gestes de Benoît Lacroix constituent, dans une accumulation de détails vécus, de formules heureuses et de raccourcis saisissants, le meilleur portrait de Benoît Lacroix, complété par une chronologie de sa vie, une bibliographie de ses œuvres et des pages autobiographiques pleines de fraîcheur et de poésie ${ }^{1}$. J'y renvoie le lecteur qui voudrait connaître davantage Benoît Lacroix ${ }^{2}$. Je me limiterai ici, après un profil rapide de sa biographie, à évoquer surtout ce qui le relie au monde de la religion populaire, selon les intérêts de notre revue.

À quatre-vingt-douze ans, Benoît Lacroix, droit comme un chêne, l'œil vif et le sourire captivant, continue à travailler. Il vient de publier deux autres volumes chez Fides. L'Université de Naples lui a consacré un congrès international (14-15 novembre 2005)3. Le réalisateur Luc Gouin prépare sur lui, sous le titre Lacroix sur parole, un documentaire biographique destiné à la télévision. Bref, Benoît Lacroix marche imperturbable vers le cap des cent ans. Je suis heureux de lui offrir ici ce témoignage, pour rapide et incomplet qu'il soit.

1. Sous la direction de Giselle Huot, Dits et gestes de Benoît Lacroix, prophète de l'amour et de l'esprit, Saint-Hippolyte, Éditions du Noroît, et Montréal, Fondation Albert-le-Grand, 1995, 735 p. Cf. «Chronologie », p. 599-651, et « Bibliographie sélective », p. 653-666.

2. Il existe aussi, disponibles souvent sur la toile, d'autres « portraits ». Le plus complet, teinté d'admiration et d'humour, est celui de Guy Laperrière, à l'adresse agora.qc.ca/encyclopedie/index.nsf/ Impression/Benoit_Lacroix.

3. L'Humanisme franco-canadien. Un cas : Benoît Lacroix, Atti del convegno internazionale, 1415 novembre 2005, a cura di Marina Zito, Napoli, Università degli studi di Napoli « l'Orientale », 2007, $191 \mathrm{p}$. 


\section{Notice biographique}

Joachim Lacroix (le nom de Benoît lui sera attribué à son entrée chez les dominicains, en souvenir de Benoît XI, un pape dominicain du moyen âge) est né à Saint-Michel-de-Bellechasse le 8 septembre 1915, d'une famille de cultivateurs. Il évoquera souvent, dans ses œuvres, la beauté grandiose des paysages de son enfance, en bordure du grand fleuve, en face de l'île d'Orléans, et prétendra que ces grands espaces l'ont immunisé contre les idéologies étriquées et les systèmes rigides. Il fit ses études secondaires au grand Collège de Sainte-Annede-la-Pocatière (1927-1936). Ce furent « des années inoubliables ».

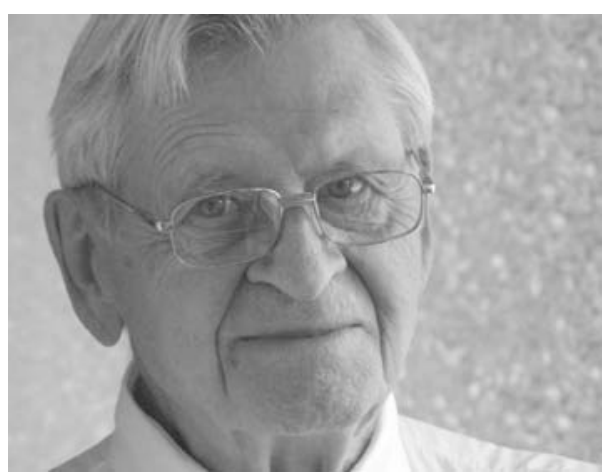

Gracieuseté de Luc Gouin, 2007.

Il se souviendra de ses professeurs comme d' «humanistes-nés », qui lui donnèrent le goût de la littérature. Ils lui donnèrent aussi, on peut supposer, cette maitrise élégante de la langue française qui le caractérise.

En juillet 1936, il entrait au noviciat des dominicains à Saint-Hyacinthe. Sa vocation fut éveillée par des missionnaires, anciens élèves du Collège, qui étaient venus y prêcher. En août 1937, il passait au Studium generale dominicain à Ottawa, pour y faire deux années de philosophie, puis deux années de théologie. « Ils m'ont converti à l'étude », dira-t-il de ses professeurs dominicains. Après son ordination sacerdotale, en 1941, on l'envoya compléter ses études théologiques au St.Basil's Seminary de Toronto (19411943). En même temps, il entreprenait au Pontifical Institute of Mediaeval Studies de Toronto des études en histoire médiévale. Ses supérieurs entendaient renforcer, par un jeune professeur formé aux meilleures écoles, l'Institut d'études médiévales qu'avait fondé au Studium Generale d'Ottawa, en 1930, le grand médiéviste français Marie-Dominique Chenu, et dont le personnel avait été fauché par la guerre. Il obtint le lectorat en théologie (septembre 1943), la licence (mai 1944) et le doctorat ès sciences médiévales (mai 1951). Il compléta ses études par des stages à l'École de Hautes Études et l'École des chartes à Paris (1952-1953), au Centre d'études supérieures de civilisation médiévale de Poitiers (1957) et enfin, muni de la prestigieuse Bourse Guggenheim, à l'Université de Harvard (1959-1960). Pendant ses années de formation, il rencontra de grandes personnalités de la culture, dont 
Étienne Gilson, Jacques Maritain, Henri-Irénée Marrou, avec qui il se lia aussi d'amitié.

En 1945, il commença son enseignement à l'Institut d'études médiévales qui, dans l'entre-temps, avait été transféré à Montréal en 1942, d'abord dans un édifice de la rue Rockland puis, à partir de 1962, dans la nouveau Couvent de Saint-Albert-le-Grand, dans la Côte-Sainte-Catherine. Ce fut dans cet Institut, rattaché à l'Université de Montréal, que Benoît Lacroix exerça pendant quarante ans son travail de professeur. Il y enseigna l'histoire médiévale, notamment l'histoire de la culture et des idées. Il en fut le directeur de 1963 à 1969. Il n'interrompit son enseignement à l'Université de Montréal que pour des séjours au Japon, professeur invité à l'Université de Kyoto (1961), à l'Université du Rwanda (1965-1966) et à l'Université de Caen (1973-76). En 1981, il quitta l'Université, non pour prendre une retraite bien méritée mais, nous dit-il, pour travailler plus librement dans «l'université élargie de tous ceux qui cherchent autrement et ailleurs ", comme auteur, animateur, prédicateur, communicateur, professeur invité, consultant. Plus qu'auparavant, peut-être, il est devenu une figure familière dans le panorama religieux et intellectuel du Québec.

Dans son rôle de professeur d'histoire médiévale, Benoît Lacroix a publié un bon nombre de monographies et de travaux érudits ${ }^{4}$. Il chérissait l'idée que la culture québécoise, en particulier la religion populaire, qui n'avait connu ni la Réforme ni la Révolution française, avait un lien particulier avec le moyen âge : « Le Canadien français aimera le moyen âge, comme on aime son enfance », ce moyen âge qui, disait-il encore, « est en chacun de nous ». Mais son inépuisable activité a touché à bien d'autres domaines. Il s'est intéressé très tôt à la littérature québécoise, qu'il a voulu faire connaître et valoriser. Il a composé lui-même des œuvres de fantaisie (Le P'tit Train, Les Cloches et bien d'autres), d'une facture délicieuse. Il a été le collaborateur, conseiller et ami d'écrivains et poètes. Avec Jacques Brault, il a entrepris la monumentale édition des œuvres de Saint-Denys Garneau. Dans le domaine de l'histoire du Québec, il a été l'animateur et le soutien indéfectible de l'édition des œuvres inédites de Lionel Groulx (journal, correspondance), et publié sur lui plusieurs travaux. Il fut un infatigable promoteur dans le domaine de l'édition: membre fondateur de la collection "Classiques canadiens » aux éditions Fides de Montréal (1955-1972), directeur-fondateur de la collection « Vie des lettres canadiennes » aux Presses de l'Université Laval (1956-1975), fondateur des Cahiers d'histoire du Québec au XX' siècle (1993), cofondateur des Cahiers de Saint-Denys Garneau (1996), membre fondateur,

4. Les références aux publications évoquées dans l'article sont données en appendice, dans la Bibliographie. 
avec Fernand Dumont, de l'Institut québécois de recherche sur la culture. Proche de la revue Maintenant, des pères Georges-Henri Lévesque et Henri Bradet (« un des pères douloureux de notre Révolution tranquille »), Benoît Lacroix a aussi publié un grand nombre d'essais sur les problèmes culturels et sociaux du Québec. On ne saurait oublier, enfin, son rôle de guide spirituel, de prédicateur, d'animateur de groupes religieux, de commentateur dans les médias. Benoît Lacroix attache une importance primordiale à cet aspect de son activité ("Mon état de dominicain est mon plus beau titre de gloire», a-t-il dit souvent), et y a consacré un grand nombre de publications. Il aura été, a-t-on dit, « le directeur spirituel du Québec » (Jacques Grand'Maison), « le conseiller, l'inspirateur et l'ami des professionnels du monde des médias » (cardinal Turcotte).

Dans une carrière si longue et intense, Benoît Lacroix a reçu nombre de témoignages officiels d'estime. Il n'a jamais eu la vanité de les chercher, ni la coquetterie de les refuser. Il est membre de la Société royale du Canada (1971), membre de l'Académie des sciences morales et politiques (1971), Prix Gérin-Lajoie (1981), membre de la Société des Dix (1982), Grand Officier de l'Ordre du Canada (1985), Docteur honoris causa de l'Université de Sherbrooke (1990), Chevalier de l'Ordre national du Québec, Grand Officier de l'Ordre national du Québec (1996), récipiendaire de prix et médailles en grand nombre.

\section{Benoît Lacroix et la religion populaire}

L'intérêt pour la religion populaire comme champ privilégié de recherche apparaît chez Benoît Lacroix dans la dernière phase de sa carrière universitaire, vers les années 1965-1970. Les raisons qui peuvent expliquer cette évolution tiennent autant au personnage lui-même qu'à des traits conjoncturels plus généraux ${ }^{5}$.

Dans son expérience de directeur de l'Institut d'études médiévales (19631969), Benoît Lacroix avait constaté que les thématiques traditionnelles, à savoir l'histoire de la pensée philosophique et théologique du moyen âge, intéressaient de moins en moins les étudiants. Le Québec était en train de changer radicalement et les étudiants demandaient « un autre moyen âge », selon l'expression de Jacques Le Goff. C'est dans ce contexte que, à partir des années 1970 environ, on commença à entrevoir, chez le personnel et dans les programmes de l'Institut, les amorces d'intérêts nouveaux, orientés vers l'anthropologie religieuse, la vie religieuse du peuple, les mouvements

5. Je reprends ici certains éléments de mon étude « Benoît Lacroix et la religion populaire », dans L'Humanisme franco-canadien ( $c f$. note 3), p. 11-52. 
marginaux et d'autres domaines analogues. En somme, on passait de la pensée théorique à l'expérience vécue, des élites au peuple.

Chez Benoît Lacroix, ces nouvelles orientions se concrétisèrent dans un intérêt de plus en plus marqué pour la religion populaire. Des collègues et amis, canadiens et européens (Fernand Dumont, Henri-Irénée Marrou, Michel Meslin, Jean Delumeau) l'encouragèrent dans cette direction, que favorisait aussi le climat théologique de l'époque. Dans les années 1970-1975 s'était amorcé en effet, un peu partout dans l'Église catholique, une réflexion sur la religion populaire, ses modalités, ses valeurs, sa gestion. Le renouveau de l'ecclésiologie suscité par le Concile Vatican II comportait un effort pour élargir l'Église à tout le «peuple de Dieu », en dépassant l'identification privilégiée de l'Église avec le clergé. Cette « ecclésiologie du peuple de Dieu » entendait donner une dignité nouvelle à toutes les couches sociales, à toutes les formes de langage religieux. Cet effort se concrétisa dans des synodes et des conciles, des déclarations pastorales, des documents méthodologiques.

Mais l'intérêt de Benoît Lacroix pour la religion populaire avait aussi de profondes racines personnelles. En passant de la culture paysanne de Bellechasse à la théologie scolastique d'Ottawa et de Toronto, Benoît Lacroix avait subi une déculturation profonde, tout en gardant admiration et nostalgie pour le monde de son enfance. L'étude de la religion populaire a dû lui apparaître comme le moyen de refaire l'unité intérieure de sa vie. Il devint l'interprète théologique et culturel de la religion de son enfance. "Les habitants de Bellechasse constituent le lieu théologique par excellence de ma vie religieuse et sacerdotale », dira-t-il. Fernand Dumont l'admirait pour avoir su concilier de manière unique sa contribution à la culture savante et son affinité avec les gens des milieux populaires : «Cette présence aux deux extrémités de la culture, sans fausseté, sans artifice, est tout à fait exceptionnelle».

Le premier résultat de cet intérêt fut la création, en 1967, du Centre d'études des religions populaires. Ce n'était, du point de vue de l'organisation, qu'un petit local, aménagé dans le Couvent de Saint-Albert à Montréal, mais ces modestes débuts avaient le mérite de proposer, parmi les premiers, ce domaine de recherche. Le Centre publia d'abord des cahiers polycopiés, puis commença à organiser une série systématique de colloques, dont les actes furent presque tous publiés : Bilan méthodologique (Saint-Gervais-deBellechasse, 1970), Le Merveilleux (Québec, 1971), L'Imagerie populaire (Montréal, 1972), Archives et religion populaire (Sherbrooke, 1973), Foi populaire, foi savante (Ottawa, 1974), Religion populaire, milieu naturel et cadre social (Chicoutimi, 1975), Les Pèlerinages au Québec (1975), Folklore maritime et religion populaire (Moncton, 1977), Religion populaire et travail (Sudbury, 1978), Médecine populaire et religion traditionnelle (Musée de 
l'homme, Ottawa, 1980). La synthèse de ces recherches fut un grand colloque sur la religion populaire au Québec (Religion populaire, religion de clercs?, Laval, 1982). En " pionnier sauvage », selon les termes de sa collaboratrice Giselle Huot, Benoît Lacroix avait conçu le Centre justement dans cette perspective d'animation : « dans ma tête, c'était très clair : dix colloques, dix publications, et ensuite on ferme les livres. Et c'est arrivé ». Le Centre a été en effet démantelé en 1994, livres et cassettes étant remis au Centre LionelGroulx. Mais le germe avait été déposé. La venue à Montréal du grand médiéviste italien Raoul Manselli, dont le résultat fut le volume La Religion populaire au moyen âge. Problèmes de méthode et d'histoire, étude de pionnier, traduite aussi en italien, fit connaître en Europe le travail des Canadiens sur ces problèmes ${ }^{6}$.

En plus de cette activité d'animation, et nombre d'études partielles, l'apport majeur de Benoît Lacroix, arrivé tard dans sa carrière, car longuement mûri, est constitué par son témoignage personnel sur la religion populaire du Québec traditionnel. Ce témoignage, Benoît Lacroix l'a consigné dans deux œuvres originales et intenses : Le Religion de mon père (1985) et - plus complète, plus ample, plus systématique - La Foi de ma mère (1999). Il est impossible de classer ces œuvres dans un genre précis. Elles demeurent inclassables, comme Benoît Lacroix lui-même. Elles tiennent à la fois du souvenir autobiographique et du reportage ethnologique, de la micro-analyse locale et de la synthèse d'anthropologie culturelle. Caïus Lacroix et RoseAnna Blais sont un couple réel, inoubliable, mais ils sont aussi les archétypes de ces couples qui ont construit un pays : portrait d'un couple, portrait d'un peuple. Le village de Bellechasse cache en filigrane tout village québécois traditionnel.

Dans ces œuvres, Benoît Lacroix - l'intellectuel, le théologien, l'historien, le citadin - se considère et se sent toujours un villageois de Bellechasse. Il décrit en interprétant et en s'interprétant, il explicite l'implicite, il dit le nondit de cette culture. Pourtant ces considérations et ces tournures sonnent plus vraies qu'un protocole de reportage ethnographique. C'est le secret de Benoît Lacroix : sujet et objet, personnage et auteur, témoin et interprète d'une expérience biographique et locale qui se veut miroir d'une expérience nationale, une synthèse de la religion populaire du Québec. Cet extraordinaire exercice d'auto-ethnologie, ou autobiographie anthropologique, restera un témoignage précieux. Fernand Dumont le considérait comme «le travail le plus important dans ce domaine ».

6. Raoul Manselli, La Religion populaire au moyen âge. Problèmes de méthode et d'histoire, Montréal, Institut d'études médiévales, 1975, 236 p. Il soprannaturale e la religione popolare nel Medioevo, Roma, Edizioni Studium, 1985. 
Une première caractéristique de ces œuvres est la qualité du regard que Benoît Lacroix porte sur le monde de la religion populaire. C'est un regard intelligent, parfois critique, mais respectueux, voire tendre et plein d'empathie. Benoît Lacroix se situe aux antipodes de l'attitude paternaliste, condescendante, parfois méprisante, qui caractérise à cet égard tant d'hommes d'Église. Le peuple, à les entendre, ne peut être dans le domaine religieux que naïf, frustre, primitif, un peuple « enfant», face auxquels les « adultes » seraient évidemment les clercs. Rien de tel chez Benoît Lacroix. Dans chaque croyance, dans chaque geste, il sait découvrir le sens humain. Il se peut que la tendresse de son regard sur la religion populaire soit en partie la tendresse d'un fils pour ses parents : mais ses parents sont aussi ses ancêtres, son peuple, sa terre, son pays. L'amour filial aura nourri en lui l'intelligence anthropologique.

La Religion de mon père et La Foi de ma mère nous frappent aussi par l'ampleur culturelle, au sens anthopologique du mot « culture », du tableau qu'elles proposent. La religion que ces œuvres nous décrivent n'est pas un amas de " curiosités » folkloriques, mais un système cohérent de valeurs et de conduites, une véritable Weltanschauung qui couvre tous les aspects de l'existence. C'est une synthèse religieuse et culturelle, individuelle et sociale. Elle inclut l'Église et l'église, mais aussi l'espace et le temps, la nature et le travail, la famille et la communauté, la vie et la mort.

Le temps est d'abord celui de l'année liturgique, déployée comme une sorte de vaste Légende dorée réécrite pour Bellechasse. Chaque fête y est décrite dans son sens religieux, mais aussi dans ses aspects esthétiques et ses coutumes propres. Chacune possède sa particularité. À la fête des Rois « les gens de Saint-Michel sont d'accord pour donner davantage à la quête, à cause des Africains " qui n'ont rien, qui sont loin, qui ne sont pas chrétiens" ». Au Jour de l'An, c'est le rite extraordinaire de la bénédiction paternelle, qui " supplante en importance et en émotion la messe et la bénédiction d'église ». À l'Épiphanie, quand on lit l'évangile de la multiplication du vin aux noces de Cana, c'est le temps de rappeler les dangers de l'alcoolisme, et le pauvre Azarias Dumont, « mort en boisson ».

Il y a des fêtes qui relient davantage la religion au quotidien du travail et aux dangers de la vie. Il y a la Chandeleur, avec ses cierges bénits qui, « en cas de danger, de feu dans les tuyaux de la maison, de feu à la grange ou à la porcherie, font des merveilles »; la fête de Saint Blaise, « qui a la réputation de guérir les toux et les maux de gorge, les rhumatismes, les maux de barbe »; les Rogations, avec la bénédiction des grains, «à cause que nos terres ont encore plus besoin du Bon Dieu que de nos bras »; la fête des Rameaux, 
dont les branches bénites « nous protègent contre le feu, le tonnerre, et qu'en plus attirent des bénédictions spéciales sur la maison et les bâtiments ».

Cette religion embrasse aussi l'espace : les grands espaces du ciel et du fleuve, la forêt, si souvent mentionnée, et surtout la terre, lieu d'admiration et lieu d'enracinement humain, terre qui est pour le paysan « le lieu premier de sa spiritualité ». Il y a les espaces ordinaires de la vie quotidienne. Il y a la maison de Rose-Anna, avec bénitiers, crucifix, niches, statuettes et images saintes partout. Il y a la grange, le hangar, le poulailler, les remises, avec pour chaque objet « la petite prière qui va avec». Il y a l'espace social : la paroisse, « petite patrie » faite de solidarité et d'enracinement, mais aussi de disputes, de placotages, de luttes de clocher, de querelles de voisins, de rivalités autour des places de banc. Pour Caïus Lacroix, la paroisse est la véritable patrie, « son vrai pays », celui où les ancêtres ont planté leurs croix, tracé des rangs et fixé leurs pagées.

Jusqu'à quel point peut-on penser que cette religion n'est, à tout prendre, que la religion des clercs? Qu'elle n'a aucune autonomie ? La réponse qui se dégage de ces deux œuvres est nuancée. Certes la présence du curé est écrasante. On ne discute pas l'autorité du clergé, ni les grandes obligations imposées par la religion : aller à la messe, aller à la confesse, faire ses Pâques, se marier à l'église et selon les lois de l'Église. Mais il me semble que, lorsqu'on a souligné et reconnu cette dépendance de la religion populaire des paysans québécois par rapport à la religion officielle, on n'a pas tout dit. Subtilement, mais réellement, Caïus Lacroix, plus sans doute que Rose-Anna (il y a une différence entre leur « religion populaire » respective !) revendique son autonomie, sa propre interprétation du christianisme. Son credo est fort différent du symbole des apôtres qu'il récite pourtant tous les dimanches : «mon père croyait fermement en Dieu, à la Vierge de Lourdes, au diable, aux âmes du Purgatoire, aux feux follets et beaucoup à son Bon Ange ». Sa Trinité n'est pas celle des théologiens et ne constitue certes pas le centre de sa religion : « C'est ben mêlâillant tout ça ! C'est bien emmêlâillant ! » Mieux vaut, alors, simplifier la religion : « ça fait que j'ai pour mon dire que j'vais prier le Bon Dieu qui est de l'autre bord, pis y a la Sainte Vierge, mon Bon Ange, pis les âmes ». Son paradis n'est pas celui de la vision béatifique. C'est une continuation concrète de la vie concrète. C'est l'autre bord, réel comme l'autre rivage du grand fleuve, et dans lequel il y a déjà ses grandsparents, ses ancêtres, ses amis, saint Pierre, la bonne sainte Anne. Et Caïus Lacroix n'est pas intéressé à aller au paradis pour contempler éternellement Dieu : «Si le ciel c'est pour rien faire, j'sus pas intéressé... J'demanderai à Saint Pierre d'ouvrir des terres, d'aller défricher, d'entailler des érables, peutêtre de garder des poules... ». Même face aux dogmes officiels, Caïus Lacroix 
garde sa liberté. Il discute, il filtre, il choisit. Tout ne lui semble pas si important. Dans la religion comme dans tout, « il y a du creyable, du fiable, du doutable, pis de l'incroyable ... ».

Tout au long de son œuvre, Benoît Lacroix s'est posé la question : cette « religion de nos pères » a-t-elle conduit le peuple québécois à sa libération ou à sa servitude ? A-t-elle été un opium aliénant, ou un facteur de progrès humain ? Il ne propose pas une réponse théorique, mais dans tout son récit cette culture rurale, centrée sur la religion, nous apparaît comme une culture intense, profonde, qui a su trouver des réponses aux grands problèmes de la vie et de la mort. Elle incarne des valeurs intériorisées avec une inébranlable conviction. Elle est sagesse et poésie. Elle comporte une sorte d'optimisme foncier, qui fait dire à Benoît Lacroix, à propos de son père : " sa foi était avant tout une confiance aux êtres et aux choses ». Quand on lit ces affirmations limpides et assurées, sur la fidélité, sur l'amour (« S'aimer, c'est sacré »), sur le destin, sur les ancêtres, on se sent en présence d'une sagesse tranquille, positive, qui est à sa manière une forme supérieure d'humanisme. La façon d'après laquelle les gens de Bellechasse traitent leurs vieux est le fruit accompli d'une haute civilisation. Et encore davantage le calme, la sagesse, la poésie avec laquelle ils accueillent la mort, dans la dernière et plus belle page de La Foi de ma mère.

La religion populaire n'a donc pas été, pour Benoît Lacroix un domaine d'érudition, une curiosité de savant. Elle a été au centre de ses intérêts intellectuels, théologiques et pastoraux. Il y a trouvé une forme d'humanisme, qu'il a essayé de nous transmettre, convaincu que « jamais nos machines à calculer et autres détecteurs de chiffres à penser ne viendront à bout de la sagesse d'un habitant de Bellechasse ». Mais s'il a trouvé cet humanisme dans la religion des villageois de Bellechasse, c'est parce que lui-même, Benoît Lacroix, a toujours proposé et vécu une conception humaniste de la religion. Il nous la révèle dans un de ses passages les plus caractéristiques, qui est aussi sans doute une description de sa vie :

$\mathrm{Au}$ fait, une religion est culturellement valable si elle se donne des raisons humaines d'exister. Si elle ne veut que le divin, elle risque de devenir opium ; si elle s'incarne, si elle va à l'humain, elle promet mieux d'être ferment, tel que le Christ l'a souhaité en choisissant le risque de vivre humainement, de lutter et de mourir pour ses amis les humains ${ }^{7}$.

7. La Religion de mon père, p. 242. 
Des raisons humaines d'exister, pour vivre humainement, parmi ses amis les humains, voilà la religion de Benoît Lacroix : religion de théologien, de pasteur, d'intellectuel et d'humaniste, indissolublement. Voilà aussi son portrait le plus vrai.

\section{Bibliographie du père Benoît Lacroix}

Benoît Lacroix a publié énormément : plus de cinquante livres (comme auteur, coauteur ou éditeur) et plusieurs centaines d'articles, de tous les niveaux (recherche érudite, vulgarisation, articles d'opinion, éditoriaux, préfaces, recensions, entrevues). $J$ 'en propose un choix très restreint, relatif aux points évoqués dans ce portrait.

\section{L'historien du moyen âge}

1950. Pourquoi aimer le moyen âge ? Montréal, L'Euvre des Tracts, $n^{\circ} 367$ (mars 1950), $16 \mathrm{p}$.

1951. L'Histoire dans l'Antiquité : florilège suivi d'une étude. Préface de Henri-Irénée Marrou. Montréal, Institut d'études médiévales, 1951, $252 \mathrm{p}$.

1965. Orose et ses idées. Paris, J. Vrin, et Montréal, Institut d'études médiévales, 1965, $235 \mathrm{p}$.

1966. «Travailleurs manuels du moyen âge roman : leur spiritualité », dans Mélanges René Crozet, Poitiers, CÉsCM, 1966, vol. 1, p. 523-529.

1971. L'Historien au moyen âge. Paris, J. Vrin, et Montréal, Institut d'études médiévales, 1971, $301 \mathrm{p}$.

1973. «Pastorale en préhistoire : un institut d'études médiévales », Communauté chrétienne, vol. 12, nº 70 (juillet-août 1973), p. 285-296.

1974. « Deus le volt! la théologie d'un cri », dans Études de civilisation médiévale, $\mathrm{IX}^{e}$-XII ${ }^{e}$ siècles : Mélanges offerts à Edmond René Labande, Poitiers, CÉSCM, 1974, p. 461-470.

\section{2. $\quad$ Littérature et histoire du Québec}

1954. Vie des lettres et histoire canadienne. Suivi d'un lexique pour servir à l'étude de notre littérature. Montréal, Éditions du Lévrier, 1954, $77 \mathrm{p}$.

Saint-Denys Garneau

1967. Saint-Denys Garneau. Textes choisis et présentés par B. Lacroix, $3^{\mathrm{e}}$ éd. Montréal, Fides, 1967 [1 $1^{\text {ère }}$ éd., 1956], 96 p. 
1971. Saint-Denys Garneau : ouvres. Texte établi, annoté et présenté par Jacques Brault et Benoît Lacroix. Montréal, Presses de l'Université de Montréal, 1971, xxvii-1320 p.

1996. Mémorial : inédits de Saint-Denys Garneau, de parents et d'amis. Conçu et réalisé par Giselle Huot et Benoît Lacroix. Saint-Hippolyte, Éditions du Noroît, 1996, 117 p., ill.

Lionel Groulx

1967. Lionel Groulx. Montréal, Fides, 1967, 96 p.

1978. "Lionel Groulx cet inconnu », Revue d'histoire de l'Amérique française, vol. 32, no 3 (1978), p. 325-346.

1984. Lionel Groulx. Journal, 1895-1911. Éd. critique de G. Huot et R. Bergeron, sous la dir. de Benoît Lacroix et al. Préface de Benoît Lacroix. Montréal, Presses de l'Université de Montréal, 1984, 2 v., xiv-1108 p., ill.

1989. «Lionel Groulx en $1930 »$, Les Cahiers des Dix, no 44 (1989), p. 199-229.

1990. Inventaire critique de la série de manuscrits (1923-1935) du fonds Lionel Groulx. Sous la dir. de Benoît Lacroix. Outremont, Fondation Lionel-Groulx, 1990, xcviii-344 p.

\section{Créations littéraires et voyages}

Créations littéraires

1964. Le P'tit Train. Ill. d'Anne-Marie Samson. Montréal, Beauchemin, 1964, 74 p. Nouvelle édition : Saint-Lambert, Éditions du Noroît, 1980.

1974. Les Cloches. Saint-Lambert, Éditions du Noroît, 1974, 70 p.

1981. Quelque part en Bellechasse. Saint-Lambert, Éditions du Noroît, 1981, 81 p.. Illustrations d'Anne-Marie Samson. Publié en France sous le titre Quelque part en Québec. Paris, Éditions du Cerf, 1982, 81 p.

1986. Trilogie en Bellechasse. Montréal, Éditions du Noroît, 1986, 222 p. Réunion des trois conte précédents.

1986. Marie de Saint-Michel. Montréal, Éditions Paulines, et Paris, Médiaspaul, 1986, 131 p., ill.

Voyages

1965. Le Japon entrevu. Montréal, Fides, 1965, 113 p. 
1966. Le Rwanda. Mille heures au pays des mille collines. Montréal, Éditions du Lévrier, 1966, 96 p.

\section{Religion populaire}

1972. Les Religions populaires. Colloque international de 1970 (SaintGervais, Québec). Éd. avec Pietro Boglioni. Québec, Presses de l’Université Laval, 1972, viii-154 p.

1979. « Au Canada français : typologie des sources », dans La Religion populaire. Actes du colloque international du CNRS, Paris, 17-19 octobre 1077. Paris, Éditions du CNRs, 1979, p. 315-323.

1979. «Le Moyen Âge et la culture populaire de la Nouvelle-France: l'exemple de la chanson » (avec Conrad Laforte), dans P. Boglioni, dir., La Culture populaire au moyen âge. Montréal, L'Aurore, 1979, p. 231-257.

1979. "Religion traditionnelle et les chansons des coureurs de bois», Revue de l'Université laurentienne, vol. 12 (1979), p. 11-42 (en coll. avec Conrad Laforte).

1979. «Quelques thèmes de la religion populaire chez le théologien Thomas d'Aquin » (avec Albert-M. Landry), dans P. Boglioni, dir., La Culture populaire au moyen âge. Montréal, L'Aurore, 1979, p. 163-181.

1980. Folklore de la mer et religion. Montréal, Leméac, 1980, 114 p., ill.

1981. "Pour l'étude de la religion populaire au Québec », Critère, $n^{\circ} 31$ (printemps 1981), p. 179-190.

1981. Les Pèlerinages au Québec (éd. avec P. Boglioni). Québec, Presses de l'Université Laval, 1981, 5 +160 p., 12 planches.

1983. "Archives familiales en Bellechasse », dans René Bouchard, dir., $L a$ Vie quotidienne au Québec. Mélanges à la mémoire de Robert-Lionel Séguin. Sillery, Presses de l'Université du Québec, 1983, p. 203-216.

1984. Religion populaire, religion de clercs? (éd. avec Jean Simard). Québec, IQRC, 1984, 444 p., ill.

1985. La Religion populaire au Québec : typologie des sources, bibliographie sélective (1900-1980). (Avec Madeleine Grammond). Québec, IQRC, 1985, 175 p.

1986. La Religion de mon père. Préface de Lucille Côté. Montréal, Bellarmin, 1986, 306 p., ill. 
1989. «Gens des terres d'en haut», dans Mémoires offerts au cardinal Louis-Albert Vachon. Québec, Université Laval, 1989, p. 238-244, suivi d'un Petit lexique médiéval en Beauce québécoise, p. 245-246.

1994. « Ce que l'étude des religions populaires m'a appris », dans P. Hurtubise et J.-M. LeBlanc, éd., Status Quaestionis, Ottawa, Université Saint-Paul, 1994, p. 15-22.

1999. La Foi de ma mère. Saint-Laurent, Bellarmin, 1999, 551 p., ill.

2002. La Foi de ma mère. La Religion de mon père. Saint-Laurent, Bellarmin, 2002, 498 p. (Reprise des principaux chapitres des deux œuvres précédentes).

2006. Mort et survie des religions. Préface de Josée Blanchette. SaintLaurent, Bellarmin, 2006, 53 p.

\section{Spiritualité et pastorale}

1947. Sainte Thérèse de Lisieux et l'histoire de son âme. Montréal, Les Éditions du Lévrier, 1947, 155 p. (sous le pseudonyme de Michel de Ladurantaye).

1961. Compagnon de Dieu. Montréal, Éditions du Lévrier, 1961, 365 p.

1985. Paroles à des religieuses (1950-1985). Introd. de Madeleine Grammond. Montréal, Fides, 1985, 254 p.

1991. Jeunes et croyants. Montréal, Éditions Paulines et Médiaspaul, 1991, $93 \mathrm{p}$.

1994. Le Cantique des Cantiques et son interprétation (avec Albert Carpentier). Montréal, Éditions du Noroît, 1994, 77 p.

2002. Alzheimer et spiritualité. Sous la dir. de Benoît Lacroix, avec la collaboration de Denise Lallich et d'Édith Fournier. Saint-Laurent, Fides, 2002, 76 p.

2006. Nous les vieux. Dialogue sur la vie et les choses. Propos recueillis entre Marguerite Lescop et Benoît Lacroix par François Lescop. SaintLaurent, Fides, 2006.

2007. Saint Dominique : au cœur d'une chrétienté en crise. Saint-Laurent, Fides, 2007, 165 p. 